Chapter 8

\title{
Accumulation of DNA Methylation Changes in the Progression of Gastritis to Gastric Cancer
}

\author{
Zheming Lu and Dajun Deng \\ Additional information is available at the end of the chapter \\ http://dx.doi.org/10.5772/53147
}

\section{Introduction}

The field of epigenetics describes information transmission through cell divisions of heritable changes of gene transcription activity without DNA sequence changes. Epigenetic information is biologically important for tissue or organ development and cell differentiation. Alteration of epigenetic information is involved in the development of cancers and other diseases. DNA methylation, histone modifications, and transmitted chromatin structure are the underlying mechanisms for epigenetic transmission. Aberrant DNA methylation is found in two distinct forms, hypermethylation and hypomethylation. Global hypomethylation and regional hypermethylation are characterized as two features of human cancer cells [1,2].

The regional hypermethylation involves CpG islands located in the promoter and 5'-exon(s). DNA methylation, the incorporation of a methyl group to the C-5 position of the cytosine ring in the context of 5'-CpG-3' dinucleotides, which leads to the formation of 5-methylcytosine (5-mC), is the most studied epigenetic change to date in gastric carcinogenesis. Hypermethylation of CPG islands recruits methyl DNA binding proteins, and subsequently histone deacetylases. Deacetylation of the histone tails makes the DNA structure of the promoter into a closed chromatin structure that is inaccessible to transcription factors leading to transcriptional silencing of tumor suppressor genes, mimicking their genetic mutations. Thus, aberrant hypermethylation of these CpG islands acts as an alternative way to genetic changes for the inactivation of tumor suppressor genes. Global hypomethylation at repetitive sequences causes genomic instability. Both types of DNA methylation changes were implicated in the development and progression of cancers [1-3].

It is interesting to note that site-specific gene hypermethylation is an early event in $H$. pylori -related gastric carcinogenesis. Kang et al. [4] indicated that gastric cancer (GC) as well as its associated normal mucosa exhibited the highest number of methylated genes when com- 
pared the tissues from other organs, including the lung, breast, colon, and liver (Fig. 1). This suggests the possibility that aberrant hypermethylation of $\mathrm{CpG}$ islands is more involved in the carcinogenesis in the stomach than in the other human tissues or organs. Methylation changes of some CpG islands can be detected in cancer tissues and morphologically normal gastric tissues from patients with GC or precancerous lesions, including epithelium dysplasia and intestinal metaplasia, but cannot be observed in gastritis lesions or normal gastric tissues from subjects without malignant disease.
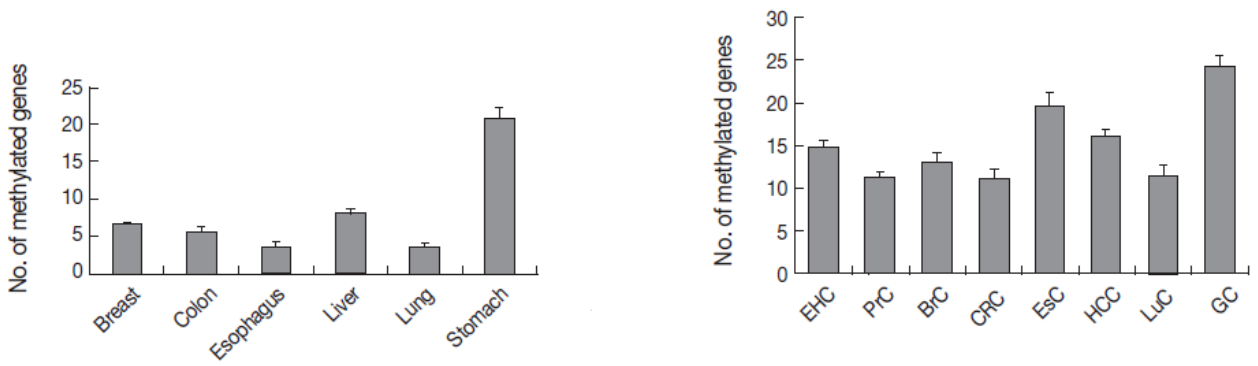

Figure 1. Bar graphs display the number of methylated genes in cancer-associated normal tissues (A) and cancer tissues (B). [4] The error bars indicate the standard error of the mean. Forty-one genes are analyzed for their methylation status in tissue samples from eight human cancer tissue types and six types of cancer-associated normal tissue using the MethyLight assay. EHC, extrahepatic bile duct cancer; PrC, prostate adenocarcinoma; BrC, breast adenocarcinoma; CRC, colorectal adenocarcinoma; EsC, esophageal adenocarcinoma; HCC, hepatocellular carcinoma; LuC, lung adenocarcinoma; GC, gastric adenocarcinoma.

\section{Accumulation of DNA methylation changes in progressions of gastritis, intestinal metaplasia, and dysplasia to GC}

As early as in the 19th century, a German pathologist von Waldeyer stated that 'cancer, in particular gastric cancer, may be considered an adaptive response to an adverse environment, characterized by a progressive phenotypic alteration.' Currently, it is well accepted that the phenotypic transformation preceding GC, that is, superficial gastritis, chronic atrophic gastritis (CAG), intestinal metaplasia, and dysplasia/adenoma, are the consequences of an accumulation of molecular alterations triggered by a chronic inflammatory process $[5,6]$. Therefore, the progressive phenotypic alteration could at times represent an altered epigenome, which is the result of host adaptive responses to environmental exposure. If hypermethylation of promoter $\mathrm{CPG}$ islands of some genes plays an important role in the malignant transformation of gastric epithelial cells, it's reasonable to assume that the pattern of hypermethylation could be found in premalignant lesions in the stomach. Kang et al. investigated the methylation profile in multistep lesions of the stomach and determined the methylation frequency of 12 genes, including APC, COX-2,DAP-kinase, E-cadherin, GSTP1, hMLH1, MGMT, p16, p14, RASSF1A, THBS1, and TIMP3, by methylation-specific PCR.They 
demonstrated that hypermethylation of certain promoter CpG islands occurs early in gastric carcinogenesis and accumulates during progression of the gastric lesion along the multistep carcinogenesis [7-9]. Tables 1 lists the methylation frequencies of tested CpG islands. The most tested $\mathrm{CpG}$ islands exhibited increase methylation tendency along the progression, such as COX-2, DAP-kinase, GSTP1, hMLH1, p14, p16, RASSF1A, THBS1, and TIMP-3. The average number of methylated genes was 2.7, 3.6, 3.4, and 5.2 per 12 tested genes in CG, IM, GA, and GC, respectively. Methylation index was defined as ratio of the number of methylated genes to the number of total tested genes. The average methylation index was $0.23,0.3$, 0.28 , and 0.43 in chronic gastritis, intestinal metaplasia, adenoma, and cancer, respectively. The methylation index was significantly higher for precancerous lesions intestinal metaplasia and adenoma than for gastritis. A significant increase of methylation frequency was observed from these pre-malignant lesions to gastric cancer [8].

\begin{tabular}{|c|c|c|c|c|c|}
\hline & $C G(N=74)(\%)$ & $\mathrm{IM}(\mathrm{N}=57)(\%)$ & $\mathrm{GA}(\mathrm{N}=79)(\%)$ & $\mathrm{GC}(\mathrm{N}=80)(\%)$ & $p$ value \\
\hline APC & $48(64.9)$ & $46(80.7)$ & $57(72.2)$ & $62(77.5)$ & $N S^{a}$ \\
\hline $\operatorname{cox} 2$ & $1(2.2)$ & $5(8.8)$ & $3(3.8)$ & $37(46.3)$ & $<0.001$ \\
\hline DAP-K & $26(35.1)$ & $28(49.1)$ & $27(34.2)$ & $45(56.3)$ & 0.012 \\
\hline E-cadherin & $63(85.1)$ & $41(71.9)$ & $46(58.2)$ & $53(67.5)$ & 0.003 \\
\hline GSTP1 & 0 & 0 & 0 & $13(16.3)$ & $<0.001$ \\
\hline hMLH1 & 0 & $4(7)$ & $7(8.9)$ & $16(20)$ & $<0.001$ \\
\hline MGMT & $11(14.9)$ & $5(8.8)$ & $8(10.1)$ & $17(21.3)$ & NS \\
\hline p14 & $22(29.7)$ & $18(31.6)$ & $60(75.9)$ & $50(62.5)$ & $<0.001$ \\
\hline p16 & $2(2.7)$ & $4(7)$ & $9(11.4)$ & $35(43.8)$ & $<0.001$ \\
\hline RASSF1A & 0 & 0 & 0 & $6(7.5)$ & 0.001 \\
\hline THBS1 & $13(17.6)$ & $28(49.1)$ & $27(34.2)$ & $45(56.3)$ & $<0.001$ \\
\hline TIMP3 & $17(23)$ & $25(43.9)$ & $22(27.8)$ & $52(65)$ & $<0.001$ \\
\hline $\begin{array}{l}\text { Average number of } \\
\text { methylated genes }\end{array}$ & $2.7^{b}$ & $3.6^{c, d}$ & $3.4^{\mathrm{b}, \mathrm{c}}$ & $5.2^{\mathrm{d}}$ & \\
\hline methylation index & 0.23 & 0.3 & 0.28 & 0.43 & \\
\hline
\end{tabular}

aNot significant $(p>0.05)$.

${ }^{\mathrm{b}}$ Chronic gastritis versus gastric adenoma; $p=0.034$; Student's t test.

' Intestinal metaplasia versus gastric adenoma; $p=0.527$; Student's t test.

${ }^{d}$ Intestinal metaplasia versus gastric carcinoma; $p<0.001$; Student's t test.

Table 1. Methylation Frequency of Each Gene in CG, IM, GA, and GC [8] 
During multistep gastric carcinogenesis, there is a steep rise in the number of methylated genes from chronic gastritis to intestinal metaplasia, which was a consistent finding in a series of studies. Intestinal metaplasia is a precancerous lesion with the typical characteristics of trans-differentiation of gastric progenitor cells into those committed to intestinal cell lineage, which normally present in intestinal mucosa [10]. Regardless of the status of $\mathrm{H}$. pylori infection, the number of methylated genes in chronic gastritis with intestinal metaplasia was significantly higher than that in chronic gastritis without intestinal metaplasia[9]. This suggests that intestinal metaplasia is an epigenetically altered lesion. Hypermethylation of promoter $\mathrm{CpG}$ island in chronic gastritis without intestinal metaplasia occurs in association with $H$. pylori infection [11] and aging [12,13]. It is unknown whether trans-differentiation results in these epigenetic changes.

Although the loss of DNA methylation was the first epigenetic alteration identified in cancer[14], global hypomethylation has been overlooked in favor of gene promoter associated hypermethylation. Global DNA hypomethylation is associated with hypomethylation of normally methylated repetitive sequences, such as LINE1, Alu, and Sat $\alpha$, as well as centromeres and microsatellite DNA $[15,16]$. In a recent study, using the immunohistochemical evaluation of 5-mC, Compare et al. [6] have found a gradual decrease in the global DNA methylation from $H$. pylori-negative normal gastric biopsy to $H$. pylori-positive chronic gastritis lesions, H. pylori-positive CAG, and GC tissues, which suggests that the global DNA hypomethylation could be implicated in $H$. pylori-related gastric carcinogenesis at an early stage. $H$. pylori-eradication can not reverse the global hypomethylation in precancerous lesions. This has been confirmed by assessing hypomethylation of repetitive sequences instead of by the global 5-methylcytosine content. Yoshida et al. [17] have reported increased hypomethylation of DNA repetitive elements through the multistep process of gastric carcinogenesis and suggested that $H$. pylori infection may induce hypomethylation of repetitive elements. Although the exact mechanism by which the global loss of DNA methylation contributes to the neoplastic process is unknown, it is believed that it may induce chromosomal instability and activate cellular proto-oncogenes, which leading to an increased risk of cancer. Bester et al. have reported that nucleotide deficiency promotes genomic instability in early stages of cancer development [18]. It is interesting to investigate if the nucleotide deficiency also leads to the global hypomethylation.

Indeed, hypomethylation and hypermethylation of $\mathrm{CpG}$ islands in the gene promoter region may activate proto-oncogenes or inactivate tumour suppressor genes that confer selective growth advantage leading ultimately to cell hyperproliferation and cancerous growth $[19,20]$.

\section{Dynamic DNA methylation related to progression of gastritis}

The stomach is one of the organs frequently showing aberrant methylation of $\mathrm{CpG}$ islands in epithelial cells because it is directly contacted with chemical and biological toxic agents daily. DNA methylation changes accumulate continuously during progression of gastritis, par- 
ticularly during the establishment of the methylation pattern. Dynamic changes of methylation pattern occur consistently for gastric mucosae cells adapting to the environmental causal factors such as $H$. pylori infection, high concentration of salts, and N-nitroso compounds. For example, on one hand, p16 inactivation by methylation in cancer cells is very stable. Methylation of $p 16 \mathrm{CpG}$ islands can be gradually restored within about two weeks after demethylation induced by DNMT1 inhibitor treatment [21]. On the other hand, p16 methylation in gastric tissues can be reversed after $H$. pylori eradication, which will discuss below. This fluctuation nature can be partially explained by the dynamic induction of methylation/demethylation.

\subsection{Association between methylation of $\mathrm{CpG}$ islands and environmental/dietary factors, lifestyle, aging in gastric carcinogenesis}

Despite strong evidences from observational epidemiology data and experimental animal studies suggesting that environmental/dietary factors, lifestyle, and aging are risk factors of gastric cancer, there has been limited understanding of the mechanisms through which such exposures have their effects on the molecular steps in tumorigenesis. It is now becoming apparent that altered epigenetic marks may play a fundamental role in determining not only susceptibility to cancer, but also contribute to promote neoplastic pathogenesis. Alternatively, the altered epigenome could at times represent adaptive responses to environmental exposure.

Environmental factors known to play crucial roles in the etiology of human cancer include chemical carcinogens (such as those found in cigarette smoke), microorganism infections, dietary contaminants (such as N-nitroso compounds), and lifestyles (such as alcohol consumption, excess intake of salt), deficiency of nutritional regimes. Stress may also contribute to the development of gastric cancer. Environmental and dietary factors in animals and humans inevitably affect epigenetic patterns, although a clear-cut causal relationship has yet to be established. The major obstacle in establishing such relationship is the fact that environmental and dietary factors induce changes are most likely subtle and cumulative, and culminate into a quantitative manifestation over a long period of time.

While animal and human studies have linked dietary factors to epigenetic regulation, it has been challenging to determine the exact mechanisms that nutrients may systemically affect epigenetic changes. The most studied and best-understood fact is the relationship between dietary methyl donors (including vitamins B6 and B12, methionine, and folate) and DNA methylation [22]. As an essential amino acid, methionine plays the central role in the epigenetic regulation by serving as a methyl donor for methylation reactions. In the process of cytosine methylation, DNMT enzyme converts the donor S-adenosyl-L-methionine (SAM) to S-adenosylhomocysteine (SAH), and transfers a methyl group from the donor SAM to the C-5 cytosine carbon atom. Therefore, an optimal supply of SAM or removal of SAH is essential for a normal establishment of genome-wide DNA methylation patterns. When methyl groups are in short supply, there is a competition for the limited resources. Perturbations in this system may be caused by dietary imbalances affecting the supply of methyl donors such as folate. 
Because humans tend to consume foods and nutrients that are highly interrelated, study of dietary patterns may have improved the power of detecting the effect of diet on DNA methylation. In humans, studies on diet and DNA methylation have yielded inconsistent findings.Animal studies have provided direct evidence that dietary factors induce changes in DNA methylation patterns. Bai et al. investigated whether p16 hypermethylation is an early and frequent event in gastric carcinogenesis induced by $\mathrm{N}$-methyl-N'-nitro-N-nitrosoguanidine (MNNG).The frequency and timing of $p 16$ hypermethylation during the multistep gastric carcinogenesis in Wistar rats were analyzed in various microdissected gastric lesions. Our group has found that $p 16$ methylation is a frequent and early event during MNNG-induced gastric carcinogenesis of rats [23]. The frequency of $p 16$ methylation is positively correlated with the severity of pathological abnormality of mucosa and negatively correlated with the expression of P16. The $p 16$ methylation in the distal glandular stomach epithelium was higher than that in the proximal stomach. These results suggest that hypermethylation of CpG islands may account for the silencing of $p 16$ in rat stomach and is an early event whose accumulation will finally lead to gastric carcinogenesis [23].(Table 2)

\begin{tabular}{cccc}
\hline Pathological status & Proximal & Distal & Total \\
\hline Normal & $0 / 16(0)$ & $1 / 20(5.0)$ & $1 / 36(2.8)$ \\
\hline Chronic atrophic gastritis & $3 / 20(15.0)$ & $1 / 4(25.0)$ & $4 / 24(16.7)$ \\
\hline Dysplasia & $3 / 13(23.1)$ & $6 / 11(54.5)$ & $9 / 24(37.5)^{\mathrm{a}}$ \\
\hline Adenoma II & $4 / 6(66.7)$ & $11 / 17(64.7)$ & $15 / 23(65.0)^{\mathrm{a}}$ \\
\hline Adenoma I & $4 / 6(66.7)$ & $10 / 14(71.4)$ & $14 / 20(70.0)^{\mathrm{a}, \mathrm{b}}$ \\
\hline Adenocarcinoma & $5 / 5(100)$ & $18 / 22(81.8)$ & $23 / 27(85.2)^{\mathrm{a}, \mathrm{b}, \mathrm{c}}$ \\
\hline Total & $19 / 66(28.8)$ & $47 / 88(53.4)^{\mathrm{d}}$ & \\
\hline
\end{tabular}

${ }^{a}$ Normal vs. dysplasia, adenoma-I/II, and adenocarcinoma, $\mathrm{P}<0.001$.

${ }^{b}$ Chronic atrophic gastritis vs. adenoma-l and adenocarcinoma, $\mathrm{P}<0.001$.

'Dysplasia vs. adenocarcinoma, $\mathrm{P}<0.002$

dProximal vs. distal, $\mathrm{P}<0.001$.

Table 2. Frequency (\%) of p16 methylation in tumors and various precancerous tissues of rat glandular stomach by MNNG [23]

Promoter methylation is also present in non-neoplastic cells as an age-related tissue-specific phenomenon. Aging was first revealed to be an methylation inducing factor of $E R$ methylation in colonic mucosae [24]. The correlation between high incidence of gastric cancers in older people and high methylation frequency of $\mathrm{CpG}$ islands suggests the link of aging and cancer via increased methylation changes of CpG islands. Waki et al. [12] found that methylation of E-cadherin and $p 16$ was not seen in non-neoplastic cells of organs from people $(\mathrm{n}=6$; $\leq 22$ years old). In contrast, these genes were methylated in non-neoplastic gastric epithelial 
cells of persons ( $\mathrm{n}=14 ; \geq 45$ years old) $86 \%$ and $29 \%$, respectively. Considering that non-neoplastic gastric mucosae from GC patients harbor higher methylation levels of CpG islands than gastric mucosae from control patients without GC, these non-cancer subjects with high methylation levels of CPG islands are expected to be at increased risk of GC. The finding that the stomach is one of the organs with a high frequency of aging-related methylation is consistent with the finding that GC is one of the tumors with a high frequency of CpG island methylation. The causes for high frequency of DNA methylation in the stomach may be related to the accumulation of the gastric mucosa to exogenous agents or a set of programmed aging events.

\subsection{Induction of aberrant DNA methylation in gastric mucosa by $\mathrm{H}$. pylori infection}

Up to $80 \%$ of GC patients have a current or past H. pylori infection [25]. World Health Organization has designated H. pylori as a human class I carcinogen for gastric malignancy. Although the exact mechanism of $H$. pylori-associated gastric carcinogenesis is still elusive, long-standing bacterial infection, perpetuated chronic inflammation, and sustained mucosal epithelial cell proliferation are thought to produce a carcinogenic environment which gradually causes epigenetic reprogramming of host cells in the stomach. Chan et al. [11] were the first to demonstrate $H$. pylori-associated hypermethylation in the gastric epithelia, which was consist with a recent study established the correlation between $H$. pylori infection and high levels of aberrant DNA methylation in gastric cancer. Maekita et al. [26] compared methylation levels in the non-cancerous gastric mucosae of GC and non-cancer patients with and without $H$. pylori infection, respectively. They found that methylation levels of several CpG islands in H. pylori-positive individuals were 5.4- to 303-fold higher than those in $\mathrm{H}$. pylori-negative individuals [27]. They also found that, among H. pylori-negative individuals, methylation levels were 2.2- to 32-fold higher in the non-cancerous gastric mucosae of GC patients than in the normal gastric mucosa of healthy individuals. (Fig. 2)

The fraction of methylated DNA molecules was quantified for eight regions of seven genes using DNA from the antral noncancerous gastric mucosae. Methylation levels increased in individuals with H. pylori infection whether or not they had gastric cancer. When methylation levels were compared among individuals without $H$. pylori infection, they were clearly higher in gastric cancer patients than in healthy volunteers. Error bars: standard errors

Our group studied the $p 16$ methylation among 920 subjects with gastric lesions and its association with $H$. pylori infection. The frequency of $p 16$ methylation was significantly higher in $H$. pylori positive subjects than $H$. pylori negative subjects in each category of gastric lesion. Compared with $H$. pylori negative, the odd ratios (ORs) of $p 16$ methylation were markedly elevated in H. pylori positive subjects for superficial gastritis (OR, 9.45; 95\% confidence interval [CI]: 2.94-30.41), chronic atrophic gastritis (OR, 15.92; 95\%CI: 7.60-33.36), intestinal metaplasia (OR, 4.46; 95\%CI: 2.44-8.13), indefinite dysplasia (OR, 3.67; 95\%CI:,1.90-7.10), and dysplasia (OR, 2.48; 95\%CI: 1.02-5.99).(table 4) Moreover, the frequencies of $p 16$ methylation increased steadily with the H. pylori density in gastric mucosa [28]. (table 3) 


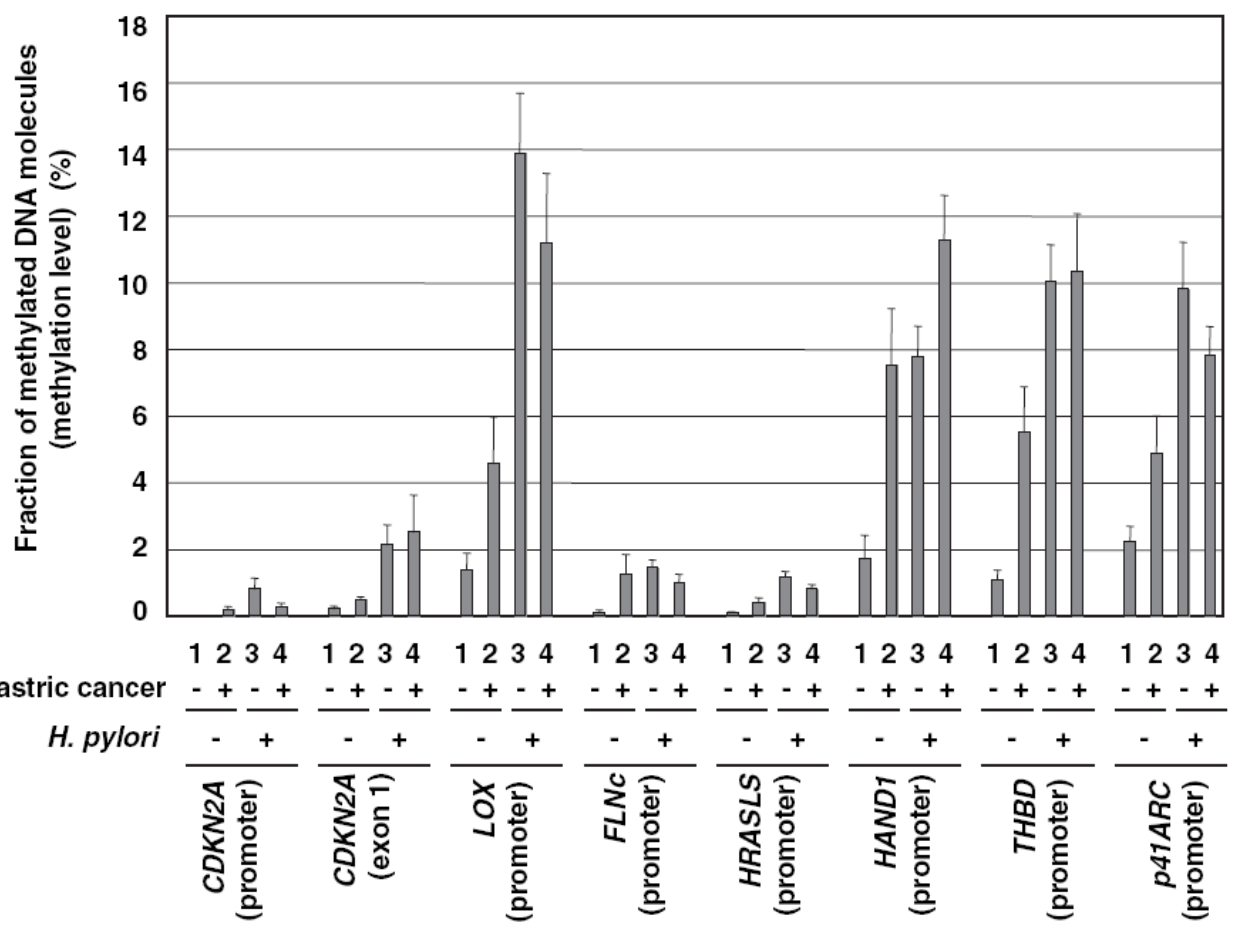

Figure 2. Methylation levels in the noncancerous gastric mucosae of individuals with and without H. pylori infection, and with and without gastric cancer[27].

\begin{tabular}{ccccc}
\hline Pathological category & H.pylori status & Methylation frequency of $p 16$ & $p_{\text {value }}$ & OR $^{2}(95 \% \mathrm{Cl})$ \\
\hline SG & No & $18.6 \%(11 / 59)$ & $<0.001$ & 1.00 \\
\hline Yes & $68.4 \%(13 / 19)$ & & $9.45(2.94-30.41)$ \\
\hline CAG & No & $21.5 \%(14 / 65)$ & $<0.001$ & 1.00 \\
\hline YM & Nes & $81.8 \%(108 / 132)$ & & $15.92(7.60-33.36)$ \\
\hline Ind DYS & Yes & $57.0 \%(81 / 142)$ & $<0.001$ & 1.00 \\
\hline No & $36.7 \%(18 / 49)$ & $<0.001$ & 1.00 \\
\hline DYS & Yes & $67.4 \%(145 / 215)$ & & $3.67(1.90-7.10)$ \\
\hline & No & $24.3 \%(9 / 37)$ & $<0.001$ & 1.00 \\
\hline
\end{tabular}

${ }^{1}$ Pearson's $\mathrm{x}^{2}$ test.-2adjusted for gender, age, smoking and drinking.

Table 3. p16 Methylation Associated With H. Pylori Infection by Paathological Category [28] 
Eradiation of $H$. pylori infection could not reverse all epigenetic changes in gastric mucosae. Leung et al. [29] have reported that one year after H. pylori eradication, there is a marked reduction in the mean methylation density of the $C D H 1$ gene in both the promoter region and exon-1. Perri et al. [30] have observed partial regression of epigenetic events one year after successfully eradicating of $H$. pylori infection therapy. CDH1, p16, and APC methylation significantly decrease while COX2 methylation completely disappear. Moreover, an animal model was adopted to strengthen these findings. H. pylori infection is best modeled in Mongolian gerbils. The incidence of gastric cancers in gerbils depends on the duration of $H$. pylori infection, and eradication of $H$. pylori significantly reduces the incidence [31]. Thus, this model is expected to be useful in analyzing detailed molecular mechanisms for induction of aberrant DNA methylation.Mongolian gerbils experimentally infected with $H$. pylori and subsequent genome-wide screening exhibited hypermethylation at several CpG islands in the gastric mucosae. Methylation levels started to increase 5-10 weeks after the infection, reached high levels by 50 weeks. Clearance of the infection resulted in markedly decreased methylation levels 10 and 20 weeks later, but they remained elevated compared with that in non-infected animals $[32,33]$. Thus, it is strongly in support of the potential beneficial effects of $H$. pylori eradication on the reversal of promoter hypermethylation in the non-cancerous stomach and possibly on chemoprevention of GCs. These findings also suggest that some methylation changes induced by H. pylori infection are temporary and some of them are persistent which is not dependent on consistent $H$. pylori infection.

The dynamic and reversible nature of methylation profile could be explained by an old concept that stem cells may be the cellular origin of cancer. Although it is unknown which methylation changes in the genome are reversible or not, one of the possible mechanisms for the diverse fates of methylation of $\mathrm{CpG}$ islands is that the fates of methylation is dependent on the fates of host cells in which the methylation occurs. The aberrant methylations are likely temporary events if they occur in fully or partially differentiated epithelial cells, because most gastric epithelial cells will quickly drop off from the gastric epithelium through regular regeneration within several days. The methylation changes are likely permanent if they occur in tissue stem cells, because these cells will remain for life-time in the stomach and even benefit from these changes. It is reported that the temporary component is the one that disappears after eradicating the infection [34-36]. Further works have demonstrated the existence of gene specificity in the DNA hypermethylation induced by infection. Methylation of specific genes that occurs in a significant number of cells in the mucosa establishes an 'epigenetic field for cancerisation' or 'epigenetic field defect', characterizing a site with high risk for subsequent malignant transformation $[33,37]$.

Although the mechanistic understanding of the H. pylori-induced alteration of DNA methylation is limited, one plausible explanation is mediated by interleukin-1 beta (IL-1 ). The level of IL-1 $\beta$ in gastric mucosa is up-regulated in specimens infected with both cagA positive and negative H. pylori strains [38,39]. Furthermore, Hmadcha et al. [40] have found that IL-1 $\beta$, through the production of nitric oxide (NO) and the subsequent activation of DNA 
methyltransferase, may induce $\mathrm{CpG}$ island methylation. $\mathrm{NO}$ is a gaseous free radical which can be produced intracellularly by nitric oxide synthase (NOS)-mediated oxidation of arginine or formed in the acidic stomach through the alternative nitrate-dependent NO pathway. H. pylori infection has been shown to be associated with up-regulation of inducible nitric oxide synthase (iNOS) both in vivo and in vitro[41,42]. Increased iNOS activity has been observed in both patients with chronic gastritis and GC patients [43]. In addition, IL-1 $\beta$ siRNA blocked $H$. pylori-induced methylation of the $C D H 1$ promoter $\mathrm{CpG}$ island in a gastric cancer cell line [44]. In an animal experiment by the Ushijima team, it has found that H. pylori infection induces hypermethylation of $\mathrm{CpG}$ island of candidate genes, and that the eradication leads to marked decrease of methylation levels in these genes [32]. Notably, the suppression of inflammation by treatment of the immunosuppressive drug cyclosporine, which suppresses inflammation without affecting bacterial colonization, abolishes this aberrant methylation in the candidate genes. These findings suggest that the infection-associated inflammatory process, rather than $H$. pylori itself, was responsible for the induction of the hypermethylation. However, in their subsequent gerbil study, neutrophilic inflammation caused by treatment with ethanol or $\mathrm{NaCl}$ does not induce DNA methylation in these genes, whereas chronic inflammation caused by $H$. pylori or $H$. felis infection leads to altered methylation in candidate genes. This finding suggests that it is not the inflammation itself, but rather specific types of inflammation, that are necessary for methylation induction[32].

\subsection{Spread of de novo methylation of $p 16 \mathrm{CpG}$ island from normal mucosae, gastritis, to gastric carcinoma}

It has been reported that $p 16$ methylation is very stable in cultured cancer cell lines based on its efficient recovery after the removal of a DNA methylation inhibitor treatment [21]. Transient transfection of $p 16$-specific artificial transcription factor can induce transcription of methylated $p 16$ alleles in cancer cells, but cannot induce demethylation of its CpG islands [45]. In contrast, most $p 16$ methylation in gastritis lesions is unstable and H. pylori-dependent [28, 30]. The mechanisms contributing to the difference of the stability of $p 16$ methylation between gastritis and cancer cells remains elusive. Characterization of the natural methylation pattern of $p 16 \mathrm{CpG}$ islands in human tissue samples with various pathological lesions may elucidate the mechanisms accounting for the diverse stability of $p 16$ methylation and could potentially be used to develop a tumor-specific methylation biomarker assay. We demonstrated that in normal gastric tissues, the majority of the samples either did not show CpG methylation or only contained sporadically methylated-CpG sites in the exon-1 coding-nucleosome region. In contrast, the gastritis and GC samples showed various degrees of methylation in the same region. Extensive methylation in both the promoter and the exon-1 regions was observed in the majority of the GC specimens. We propose that a methylation wave progressively extends from the $p 16$ exon- 1 coding- nucleosome to its promoter nucleosome in vivo. (Fig. 3) The overall p16 methylation profiles constructed from a panel of clinical specimens may approximate the natural extension pattern of de novo methylation of $p 16$ CpG islands in gastric carcinogenesis [46]. 


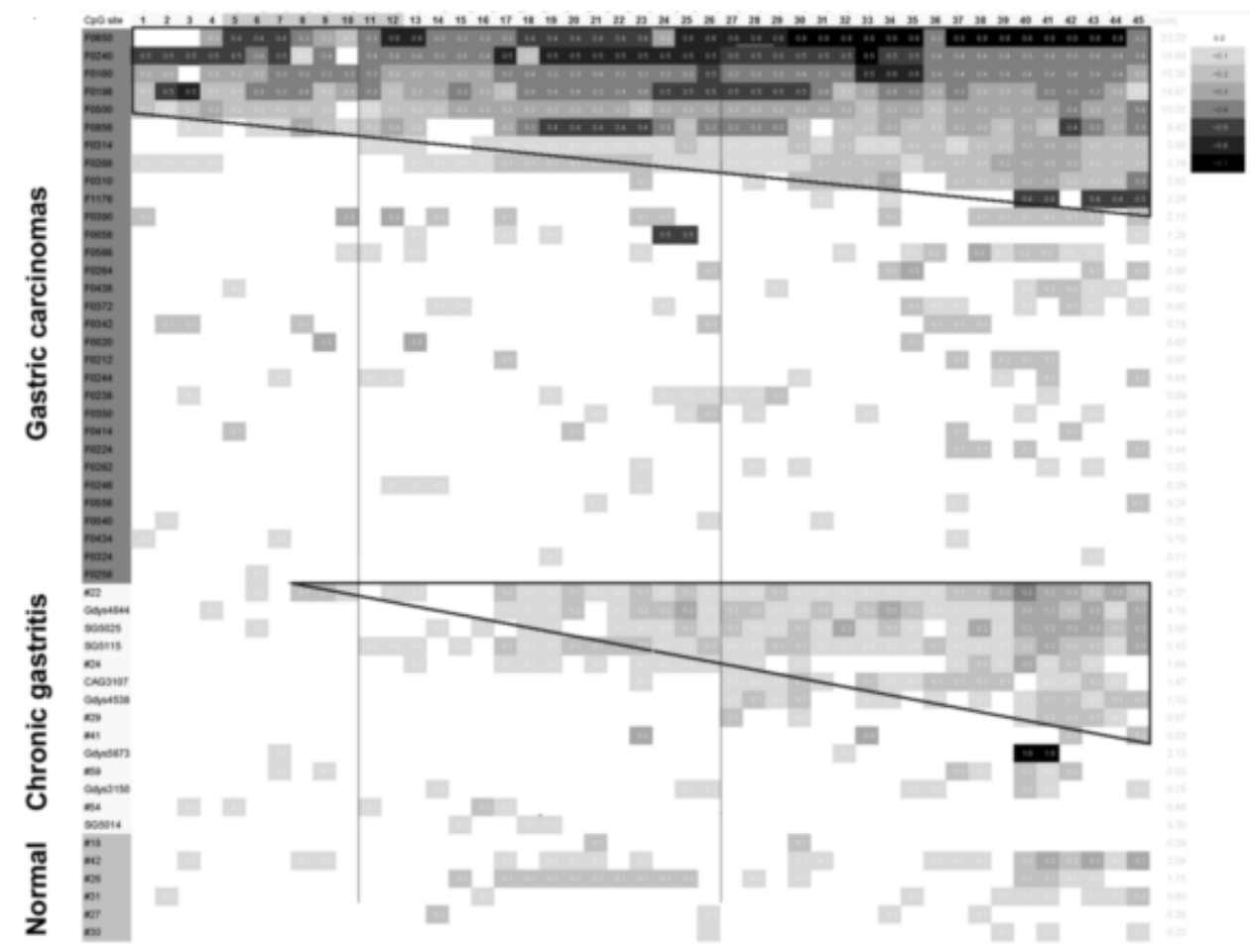

Figure 3. Comparison of methylation status of p16 CpG island in gastric carcinomas, chromic gastritis, and normal gastric mucosa [46]. A gray-graded representation of the average methylation density at individual CpG sites within the 16 promoter and exon-1 region

\section{DNA methylation as a clinical biomarker for risk assessment, and anticancer drugs target}

Epigenetic changes provide a potential explanation for how environmental factors can modify the risk for common diseases among individuals. The growing interest in cancer epigenetics stems from the fact that epigenetic changes are implicated in virtually every step of the development and progression of cancers. This is supported by the studies demonstrating that epigenetic changes including DNA hypermethylation are an early event in carcinogenesis. A distinguishing feature of epigenetic changes in comparison with genetic changes is that epigenetic changes are reversible. Therefore, aberrant DNA methylation, histone acetylation and methylation are attractive targets for cancer prevention and the epigenetic therapy. There are some evidences that methylation-based tests could predict which individuals 
are at high risk for different types of cancers, so that screening tests and prophylactic treatment can be applied more effectively. A pharmacological modification of the epigenetic status may be a potent strategy for the prevention of gastric cancers, based on the fact that methylation was a frequent event, particularly in $H$. pylori-infected patients. Perhaps methylation in normal gastric mucosa either directly predisposes the tissue to cancer or is associated with some other factors predisposing to cancer. In either case, methylation analysis of normal tissue could help determine the risk of those individuals for future development of the disease. It is reported that the genetic reduction of DNA methylation levels suppressed the incidence, number and size of gastric tumors in gastric tumorigenesis [47].

Aberrant DNA methylation is more frequently present in gastric cancers than mutations [48], so we can take advantage of the frequent presence of aberrant methylation in cancers by using it as a clue to identify tumor markers for cancer screening purposes and carcinogenic risk assessment. One advantage of using aberrant DNA methylation as a biomarker instead of mutations/expression is that it is possible to detect even a single aberrantly methylated DNA molecule embedded in 1000 unmethylated DNA molecules [49]. The high sensitivity and specificity of measurements has particular application when it is well recognized that stem cells in precancerous or cancer tissues should contribute to cancer development, metastasis, recurrence, and formation of drug resistance. In contrast, using regular gene expression assays, such as immunostaining, Western blot, RT-PCR, and Northern blot, detection of alterations of gene expression in limited number of stem cells is very difficult. Additionally, DNA methylation can be remained in various samples stored at wide-range conditions (fresh/frozen or paraffin- embedded tissue blocks, free DNA in plasma, detached cells in gastric juice, sputum, urine, stool samples, and other body fluids). For example, we observed that methylation of $p 16$, the G1 ' S conversion checkpoint gene in the cell cycle, is a powerful biomarker for prediction malignant transformation of epithelial dysplasia [50,51]. Importantly, we further developed a p16-specific probe-based quantitative MethyLight assay for the development of $p 16$ methylation diagnosis kit [52]. Developments of DNA methylation biomarkers (Septin9, p16, MGMT) for cancer screening purposes, carcinogenic risk assessment, and prediction of chemotherapy sensitivity are under clinical trials [3].

Currently, the greatest hopes are associated with clinical applications of several promising epigenetic modification inhibitors target for anticancer therapy, which inhibits DNA methylation and leads to the demethylation of the genome, thereby restoring expression of methylated genes. Such as DNMT inhibitor 5-aza-deoxycytosine and histone deacetylase inhibitors vorinostat or romidepsin, have recently approved by FDA for patients with myelodysplasia syndrome and cutaneous or peripheral T-cell lymphoma, respectively [3,53]. However, the potential pleiotropic effects of such an intervention as a result of induced genome hypomethylation (leading to, e.g., genome instability) need to be carefully considered. Therefore, the basic challenge in designing potential anticancer drugs functioning at the DNA methylation level is the specific recognition of molecular targets. 


\section{Conclusion}

In conclusion, aberrant CPG islands methylation occurs in the early stages of gastric carcinogenesis and tends to increase as the multistep process advances. It is highly plausible that $H$. pylori infection and other environment exposure can lead to the adaptive responses of the host cells to the chronic inflammation triggers the development of these epigenetic changes in the non-cancerous stomach, which eventually lead to neoplastic transformation. The epigenetic profile is highly dynamic and reversible, and varies among cells in the same organism and, in the same cell, between various states, such as health versus disease, or in response to environmental perturbations. Given the plasticity of epigenetic marks in response to cancer-related exposures, such epigenetic marks are attractive candidates for the development of surrogate endpoints which could be used in dietary or lifestyle intervention. In recent years, a lot has been learnt, but much remains to be learnt to combat gastric cancer, one of the most frequent and lethal neoplasias worldwide. The study of epigenetic alterations offers great potential for the identification of biomarkers that can be used to detect and diagnose cancer in its earliest stages, to accurately assess individual risk and as targets for chemotherapeutic strategies.

\section{Author details}

Zheming $\mathrm{Lu}^{*}$ and Dajun Deng

*Address all correspondence to: zheminglu@163.com

Key Laboratory of Carcinogenesis and Translational Research, Ministry of Education, Division of Cancer Etiology, Beijing University Cancer Hospital and Institute, Beijing, China

\section{References}

[1] Esteller M. Epigenetics in cancer. N Engl J Med. 2008; 358: 1148-59.

[2] Jones PA, Baylin SB. The epigenomics of cancer. Cell. 2007;128:683-92.

[3] Deng DJ, Liu ZJ, Du YT. Epigenetic alterations as cancer diagnostic, prognostic, and predictive biomarkers. Adv Genet. 2010; 71: 125-76.

[4] Kang GH. CpG Island Hypermethylation in Gastric Carcinoma and Its Premalignant Lesions. Korean J Pathol. 2012; 46:1-9

[5] Hatakeyama M, Brzozowski T. Pathogenesis of Helicobacter pylori infection. Helicobacter. 2006; 11(Suppl 1): 14-20.

[6] Compare D, Rocco A, Liguori E, D'Armiento FP, Persico G, Masone S, Coppola-Bottazzi E, Suriani R, Romano M, Nardone G. Global DNA hypomethylation is an early 
event in Helicobacter pylori-related gastric carcinogenesis. J Clin Pathol. 2011; 64: 677-82.

[7] Kang GH, Shim YH, Jung HY, Kim WH, Ro JY, Rhyu MG. CpG island methylation in premalignant stages of gastric carcinoma. Cancer Res 2001; 61: 2847-51.

[8] Kang GH, Lee S, Kim JS, Jung HY. Profile of aberrant CpG island methylation along multistep gastric carcinogenesis. Lab Invest. 2003a; 83: 519-26.

[9] Park SY, Yoo EJ, Cho NY, Kim N, Kang GH. Comparison of CpG island hypermethylation and repetitive DNA hypomethylation in premalignant stages of gastric cancer, stratified for Helicobacter pylori infection. J Pathol 2009; 219: 410-6.

[10] Kuipers EJ, Perez-Perez GI, Meuwissen SGM, Blaser MJ. Helicobacter pylori and atrophic gastritis: importance of the cagA status. J Natl Cancer Inst. 1995; 87: 1777-80

[11] Chan AO, Lam SK, Wong BC, Wong WM, Yuen MF, Yeung YH, Hui WM, Rashid A, Kwong YL. Promoter methylation of E-cadherin gene in gastric mucosa associated with Helicobacter pylori infection and in gastric cancer. Gut. 2003; 52: 502-6.

[12] Waki T, Tamura G, Tsuchiya T, Sato K, Nishizuka S, Motoyama T. Promoter methylation status of E-cadherin, hMLH1, and p16 genes in nonneoplastic gastric epithelia. Am J Pathol 2002; 161: 399-403.

[13] Kang GH, Lee HJ, Hwang KS, Lee S, Kim JH, Kim JS. Aberrant CpG island hypermethylation of chronic gastritis, in relation to aging, gender, intestinal metaplasia, and chronic inflammation. Am J Pathol 2003b; 163: 1551-6.

[14] Feinberg AP and Vogelstein B. Hypomethylation distinguishes genes of some human cancers from their normal counterparts. Nature. 1983; 301: 89-92.

[15] Rollins RA, Haghighi F, Edwards JR, Das R, Zhang MQ, Ju J, Bestor TH. Large-scale structure of genomic methylation patterns.Genome Res. 2006; 16: 157-63

[16] Xiang S, Liu Z, Zhang B, Zhou J, Zhu B, Ji J, Deng DJ. Methylation status of individual CpG sites within Alu elements in the human genome and Alu hypomethylation in gastric carcinomas. BMC Cancer. 2010; 10:44.

[17] Yoshida T, Yamashita S, Takamura-Enya T, Niwa T, Ando T, Enomoto S, Maekita T, Nakazawa K, Tatematsu M, Ichinose M, Ushijima T. Alu and Sata hypomethylation in Helicobacter pylori-infected gastric mucosae. Int J Cancer. 2011; 128:33-9

[18] Bester AC, Roniger M, Oren YS, Im MM, Sarni D, Chaoat M, Bensimon A, Zamir G, Shewach DS, Kerem B. Nucleotide deficiency promotes genomic instability in early stages of cancer development. Cell. 2011; 145: 435-46.

[19] Gonzalez-Zulueta M, Bender CM, Yang AS, Nguyen T, Beart RW, Van Tornout JM, Jones PA. Methylation of the $59 \mathrm{CpG}$ island of the p16/CDKN2 tumor suppressor gene in normal and transformed human tissues correlates with gene silencing. Cancer Res 1995; 55: 4531-5. 
[20] Little M, Wainwright B. Methylation and p16: suppressing the suppressor. Nat Med 1995;1:633-4.

[21] Egger G, Aparicio AM, Escobar SG, Jones PA. Inhibition of histone deacetylation does not block resilencing of p16 after 5-aza-2'-deoxycytidine treatment. Cancer Res, 2007, 67: 346-53

[22] Waterland, R. A. Assessing the effects of high methionine intake on DNA methylation. J. Nutr., 2006;136, 1706S-1710S.

[23] Bai H, Gu L, Zhou J, Deng D. p16 hypermethylation during gastric carcinogenesis of Wistar rats by N-methyl-N'-nitro-N-nitrosoguanidine. Mutat Res. 2003 ; 535: 73-8.

[24] Issa JP, Ottaviano YL, Celano P, Hamilton SR, Davidson NE, Baylin SB. Methylation of the oestrogen receptor $\mathrm{CpG}$ island links ageing and neoplasia in human colon. Nat Genet. 1994;7:536- 40 .

[25] Peek RM, Blaser MJ. 2002. Helicobacter pylori and gastrointestinal tract adenocarcinomas.Nat Rev Cancer. 2002;2:28-37.

[26] Maekita T, Nakazawa K, Mihara M, Nakajima T, Yanaoka K, Iguchi M, ArII K, Kaneda A, Tsukamoto T, Tatematsu M, Tamura G, Saito D, Sugimura T, Ichinose M, Ushijima T. High levels of aberrant DNA methylation in Helicobacter pylori-infected gastric mucosae and its possible association with gastric cancer risk. Clin Cancer Res. 2006; 12: 989-95.

[27] Ushijima T, Nakajima T, Maekita T. DNA methylation as a marker for the past and future.J Gastroenterol. 2006;41:401-7.

[28] Dong CX, Deng DJ, Pan KF, Zhang L, Zhang Y, Zhou J, You WC. Promoter methylation of p16 associated with Helicobacter pylori infection in precancerous gastric lesions: a population-based study. Int J Cancer. 2009; 124: 434-9.

[29] Leung WK, Man EP, Yu J, Go MY, To KF, Yamaoka Y, Cheng VY, Ng EK, Sung JJ. Effects of Helicobacter pylori eradication on methylation status of E-cadherin gene in noncancerous stomach. Clin Cancer Res. 2006; 12: 3216-21.

[30] Perri F, Cotugno R, Piepoli A, Merla A, Quitadamo M, Gentile A, Pilotto A, Annese V, Andriulli A. Aberrant DNA Methylation in Non-Neoplastic Gastric Mucosa of H. Pylori Infected Patients and Effect of Eradication, Am J Gastroenterol. 2007;102:13611371

[31] Nozaki K, Shimizu N, Ikehara Y, Inoue M, Tsukamoto T, Inada K, Tanaka H, Kumagai T, Kaminishi M, Tatematsu M.Effect of early eradication on Helicobacter pylorirelated gastric carcinogenesis in Mongolian gerbils. Cancer Sci 2003;94: 235-9.

[32] Niwa, T.T., Tsukamoto, T., Toyoda, T., Mori, A., Tanaka, H., Maekita, T., Ichinose, M., Tatematsu, M., and Ushijima, T. Inflammatory processes triggered by Helicobacter pylori infection cause aberrant DNA methylation in gastric epithelial cells. Cancer Res. 2010; 70, 1430-1440 
[33] Niwa T, Ushijima T. Induction of epigenetic alterations by chronic inflammation and its significance on carcinogenesis. Adv Genet 2010;71:41-56

[34] Nakajima T, Enomoto S, Ushijima T. DNA methylation: a marker for carcinogen exposure and cancer risk. Environ Health Prev Med. 2008;13:8-15

[35] Nakajima T, Yamashita S, Maekita T, Niwa T, Nakazawa K, Ushijima T.The presence of a methylation fingerprint of Helicobacter pylori infection in human gastric mucosae. Int J Cancer. 2009; 124: 905-10

[36] Feinberg AP, Ohlsson R, Henikoff S. The epigenetic progenitor origin of human cancer. Nat Rev Genet. 2006; 7: 21-33.

[37] Ushijima T, Hattori N. Molecular Pathways: Involvement of Helicobacter pyloriTriggered Inflammation in the Formation of an Epigenetic Field Defect, and Its Usefulness as Cancer Risk and Exposure Markers. Clin Cancer Res. 2012;18:923-9

[38] El-Omar EM, Carrington M, Chow WH, McColl KE, Bream JH, Young HA, Herrera J, Lissowska J, Yuan CC, Rothman N, Lanyon G, Martin M, Fraumeni JF Jr, Rabkin CS. Interleukin-1 $\beta$ polymorphisms associated with increased risk of gastric cancer. Nature. 2000, 404: 398-402.

[39] Yamaoka Y, Kita M, Kodama T, Sawai N, Kashima K, Imanishi J. Induction of various cytokines and development of severe mucosal inflammation by cagA gene positive Helicobacter pylori strains, Gut.1997, 41442 -451

[40] Hmadcha A, Bedoya FJ, Sobrino F, Pintado E. Methylation-dependent gene silencing induced by interleukin 1beta via nitric oxide production.J Exp Med. 1999; 190: 1595-604.

[41] Fu S, Ramanujam KS, Wong A, Fantry GT, Drachenberg CB, James SP, Meltzer SJ, Wilson KT. Increased expression and cellular localization of inducible nitric oxide synthase and cyclooxygenase 2 in Helicobacter pylori gastritis. Gastroenterology. 1999; 116: 1319-29.

[42] Watanabe S, Takagi A, Koga Y, Kamiya S, Miwa T. Helicobacter pylori induces apoptosis in gastric epithelial cells through inducible nitric oxide. J Gastroenterol Hepatol. 2000;15:168-74.

[43] Rajnakova A, Moochhala S, Goh PM, Ngoi S.Expression of nitric oxide synthase, cyclooxygenase, and p53 in different stages of human gastric cancer. Cancer Lett. 2001;172:177-85.

[44] Qian X, Huang C, Cho CH, Hui WM, Rashid A, Chan AO. E-cadherin promoter hypermethylation induced by interleukin-1beta treatment or $\mathrm{H}$. pylori infection in human gastric cancer cell lines. Cancer Lett. 2008; 263: 107-13.

[45] Zhang B, Xiang S, Zhong Q, Yin Y, Gu L, Deng D. The p16-Specific Reactivation and Inhibition of Cell Migration Through Demethylation of CpG Islands by Engineered Transcription Factors. Human Gene Therapy. 2012; 23: (doi:10.1089/hum.2012.070) 
[46] Lu ZM, Zhou J, Wang X, Guan Z, Bai H, Liu ZJ, Su N, Pan K, Ji J, Deng D. Nucleosomes correlate with in vivo progression pattern of de novo methylation of p16 CpG islands in human gastric carcinogenesis. PLoS ONE. 2012; 7(4): E35928.

[47] Tomita H, Hirata A, Yamada Y, Hata K, Oyama T, Mori H, Yamashita S, Ushijima T, Hara A.Suppressive effect of global DNA hypomethylation on gastric carcinogenesis. Carcinogenesis. 2010;31:1627-33.

[48] Ushijima T, Sasako M. Focus on gastric cancer. Cancer Cell 2004;5:121-5

[49] Laird PW. The power and the promise of DNA methylation markers. Nat Rev Cancer. 2003; 3: 253-66

[50] Sun Y, Deng D, You WC, Bai H, Zhang L, Zhou J, Shen L, Ma JL, Xie YQ, Li JY.. Methylation of p16 CpG islands associated with malignant transformation of gastric dysplasia in a population-based study. Clin Cancer Res, 2004, 10: 5087-5093

[51] Cao J, Zhou J, Gao Y, Gu L, Meng H, Liu H, Deng D. Methylation of p16 CpG island associated with malignant progression of oral epithelial dysplasia: a prospective cohort study. Clin Cancer Res. 2009; 15: 5178-83.

[52] Zhou J, Cao J, Lu Z, Liu H, Deng D. A 115-bp MethyLight assay for detection of p16 (CDKN2A) methylation as a diagnostic biomarker in human tissues. BMC Med Genet. 2011, 12: 67

[53] Dawson MA, Kouzarides T. Cancer epigenetics: from mechanism to therapy. Cell. 2012; 150: 12-27. 
